\title{
CyberParks Songs and Stories - Enriching Public Spaces with Localized Culture Heritage Material such as Digitized Songs and Stories
}

\author{
Kåre Synnes $^{1(\bowtie)}(\mathbb{D})$, Georgios Artopoulos $^{2}(\mathbb{D}$, \\ Carlos Smaniotto $\operatorname{Costa}^{3}$ D , Marluci Menezes ${ }^{4}$ (D), \\ and Gaia Redaelli ${ }^{5}$ (D) \\ ${ }^{1}$ Luleå University of Technology, Luleå, Sweden \\ Kare.Synnes@ltu.se \\ 2 The Cyprus Institute, Nicosia, Cyprus \\ g.artopoulos@cyi.ac.cy \\ ${ }^{3}$ CeiED Interdisciplinary Research Centre for Education and Development, \\ Universidade Lusófona, Lisbon, Portugal \\ smaniotto.costa@ulusofona.pt \\ ${ }^{4}$ National Laboratory for Civil Engineering - LNEC, Lisbon, Portugal \\ marluci@lnec.pt \\ 5 Politecnico di Milano, Milan, Italy \\ gaiaangelica. redaelli@polimi.it
}

\begin{abstract}
This chapter offers theoretical considerations and reflections on technological solutions that contribute to digitally supported documentation, access and reuse of localised heritage content in public spaces. It addresses immaterial cultural heritage, including informal stories that could emerge and be communicated by drawing hyperlinks between digitised assets, such as songs, images, drawings, texts and more, and not yet documented metadata, as well as augmenting interaction opportunities with interactive elements that relate to multiple media stored in databases and archives across Europe. The aim is to enable cultural heritage to be experienced in novel ways, supported by the proliferation of smartphones and ubiquitous Internet access together with new technical means for user profiling, personalisation, localisation, contextawareness and gamification. The chapter considers cyberparks as digitally enhanced public spaces for accessing and analyzing European cultural heritage and for enriching the interpretation of the past, along with theoretical ramifications and technological limitations. It identifies the capacities of a proposed digital environment together with design guidelines for interaction with cultural heritage assets in public spaces. The chapter concludes with describing a taxonomy of digital content that can be used in order to enhance association and occupation conditions of public spaces, and with discussing technological challenges associated with enriching public spaces with localized cultural heritage material.
\end{abstract}


Keywords: Geotagged cultural expressions - Digital cultural heritage · Historic urban fabric $\cdot$ Immaterial heritage $\cdot$ Contextual cultures $\cdot$

Participatory design

\section{Introduction}

The purpose of this chapter is to discuss the theoretical and technological approach of the CyberParks Songs and Stories concept, which aims at increasing the understanding of European cultures and creating an intercultural bridge to respond to the need for reflective and creative societies. It envisions to provide plural meanings and interpretations of (and on) heritage to citizens through collective and collaborative methods for semantic classification, contextualisation and augmentation of digital assets and associated metadata by means of machine learning, social analysis, gamification and crowdsourcing. The proposed method has similarities to what Kontkanen et al. (2016) used for species identification in terms of collaborative mechanisms.

To understand and inform the present by richer interpretations of the past, three cases of socio-cultural environments are selected: Fado songs and the identity of Mouraria neighbourhood in Lisbon (PT), oral traditions and expressions of the Patios of Córdoba (ES) and Sami Yoiks and storytelling in Sápmi (Laponia region, SE). The three cases are listed as Intangible Cultural Heritage by the Intergovernmental Committee for the Safeguarding of the Intangible Cultural Heritage (ICH) of UNESCO since 1996 (Sami Yoiks), 2011 (Fado) and 2012 (Patios). The three cases are subject to the tension between continuity with the traditions and changes, in particular those that transform substantially the layout of the territories wherein the specific cases of ICH have emerged. This tension undermines safeguarding and may jeopardise the continuity of the ICH. The concept does not define $\mathrm{ICH}$ as something frozen and perpetuated, which contradict the procedural nature of the production of $\mathrm{ICH}$, but as sociocultural subjects consciously embodied in a process of enriching, enhancing and transmitting it. The intergenerational continuum, and the spatio-temporal context of social activity, further impact the fluidity of heritage and identity.

\section{The Challenge of Accessing Intangible Cultural Heritage}

The concept recognises the pressing need in contemporary societies for inclusion and integration of information stored in individuals' memories to the heritage archives. Specifically understanding information regarding ICH activities as embodied by people, communities and societies, is imperative for capturing non-institutional knowledge, as well as complex semantic and conceptual knowledge, often expressed as nonverbal practices, rites or social relations (Artopoulos and Bakirtzis 2016). In Stiegler's (2003) terms, humans leave traces of their histories, although not produced intending to immediate transmit memories, they do so, for example writing, photography, phonography and cinematography. This perspective addresses two critical challenges of contemporary approaches to ICH: (a) facilitating access to knowledge stored in 
archives, collections and digital assets; and (b) exploiting the capacity of digital tools for enabling users to better interpret and understand the big data of ICH.

The three scenarios demonstrate the way this concept will attempt to respond to these challenges:

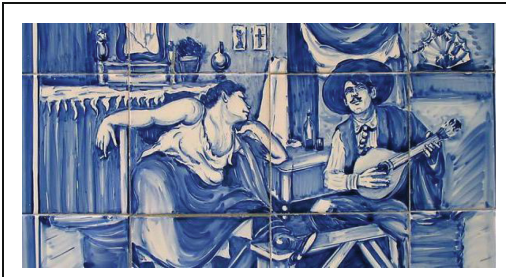

Scenario - Fado songs $\mid$ Joaquim is a member of a parish council of Mouraria, which is responsible for the development of the district. He owns a small repair shop in the Mouraria and is very involved in sharing the Fado at local 'Tasca' - a typical café or restaurant - where his grandfather sometimes plays Fado songs on a Portuguese guitar. Today Joaquim is walking home from work and passes by a mural made with the traditional Azulejo tiles, when his smartphone starts to vibrate notifying him that a point of interest is nearby. He brings up his mobile phone and the vibration becomes more frequent as he walks closer to the mural.

He stops in front of the mural and opens the mobile app to listen to the Fado song associated with it. Joaquim is reminiscent of his grandfather performing the song on his guitar, which awakes a lot of fond memories. He remembers in particular a narrative, a story that his grandfather tells at his favourite Tasca, which he decides to share with the community of Fado lovers connected through the app. Joaquim writes the short story and appends a photo of his grandfather performing the song in the Tasca. He makes a note of asking his grandfather to play it for him, so that the story can also be annotated with his performance.

Then the app suggests Joaquim to seek out similar murals associated with Fado songs located in the area. He accepts the suggestion and is presented with an annotated path along the way home. He stops at series of murals and associated places and is presented with not only other Fado songs but also with additional information such as ambient soundscapes, pictures, voice recordings and videos captured and uploaded by other users. Joaquim specifically is searching for additional information based on the Fado song played by his grandfather and when the app returns to him an image, he discovers that there are similar Fado songs, also related to the docks that were used in the past as harbours, located in the nearby neighbourhoods. Through the app he can add these locations on the map as points of interest for later, so that in his next walk around the city he can view the basins from specific vantage points that link back to his interests.

When Joaquim returns home, he uses the app to make a note on social media about his experience. Through multiple loops of this activity, shared by many, a community is formed about the docks and how to make their old uses and stories more visible to the public. Then more users of the app contribute content to this thematic group with additional narratives, effectively crowdsourcing a big amount of data related to the Fado and Mouraria. Eventually this common activity leads to the publication of articles by journalists that achieve to raise the interests of local policy makers, such as Joaquim himself, and to promote tacit arguments of the existing group of interest and researchers in the public. Finally, this action initiates social reporting activities that highlight everyday issues in Mouraria, such as destructive forms of graffiti, shortage of affordable housing and job opportunities, the degradation of built heritage, as well as the ever-growing gentrification of the area. In the appropriate context this community-building process can culminate in a process wherein people will be asking to their representatives for suitable solutions to these challenges and hence, to reviewing the existing urban policies. 


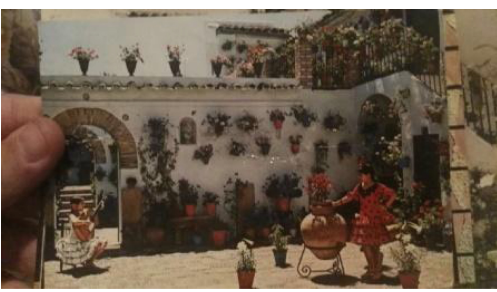

Scenario - The living patio | Maria is 92 years old and is living all her life in a patio in the district of Axerquia. There she spent her childhood, marriage and own family. She lived there with the extended family of other dwellings: they share the patio as a collective space, took care of it and in a time participated in the competition of patios. Maria's courtyard has not yet been bought by new owners, and now the houses are abandoned. Only Maria lives there, taking care of her plants and of the patio. Maria's daughter left the family home when she married in the 60's and went to live in a flat with her husband. Every year, in May, Maria's granddaughter Fuensanta returns to Córdoba to meet family and friends and "go to patios". Since Patios were declared by UNESCO ICH, the patios are attracting growing interest, in special during the Festival. This can affect the original way of living around most of the courtyards. Fuensanta arrived this year with a mobile app installed in smartphone. She uploaded some images of her grandmother's patio and other old images when they took part in the patio competition. She interviewed her grandmother about the patio, how the space was shared and the freshness of the summer thanks to shadow and vegetation. She uploaded this to the app to share it with the Patios' community. The app invites Fuensanta to explore the courtyard and to record other narratives related to her grandmother's and to her own life in the courtyard. They go to the laundry and she records her grandmother memoirs about when there was no washing machine and all the women used to wash by hand. Maria sings a song she used to sing while doing the laundry. Fuensanta uploads a photo of the current laundry and two sound records: the conversation with his grandmother and the song. Then they visit also the old communal kitchen and remember some of their favourite recipes. They look at a crockery hanging in the kitchen. They upload a photo of the crockery and hang some of the favourite recipes. The app offers diverse functions: A) to play songs uploaded by the community members; to localize in the map the sound records and the courtyards where they have been uploaded. Fuensanta discovers that there are other interviews of patio residents. B) to shows images and recipes uploaded by the community members and info about stores selling local and regional food products. Fuensanta and her sister "go to Patio" and visit some of patios, where some of their friends still live. One of them tells them that there is an association that is interested in creating in some patios of the Axerquia cooperative housing in right of use. Her friend shows her the drawing of a project for renovating the patio and Fuensanta uploads it to the application and shares it on social networks. Some people and associations interested in the patios, not as tourist product, get in contact with her. They organize a meeting inside the patio of their grandmother about how is living it, and upload photos, sound records and conclusions of the debate into the application. Many other members of the community join them, as the Instituto Andaluz del Patrimonio Historico, which includes this new information in the Atlas of the Intangible Heritage of Andalucia. The municipality and the government of Andalucia add this action in Good Practices to Safeguard Intangible Cultural Heritage and to the reports for UNESCO. Some bio-construction cooperatives and the PAX strategy get in touch with the association and Fuensanta to participate in the patio's rehabilitation and select Maria's courtyard. 


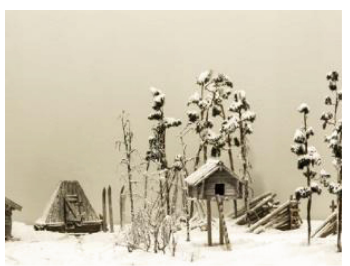

Scenario - Sámi Yoiks | Anna is 25 years old and of Sámi heritage, the only indigenous people of Scandinavia, but has as so many others of her generation left her ancestral region of Sápmi in the far north of Sweden, Norway, Finland and Russia. Anna is very aware of her Sámi heritage and wants to learn as much as possible of her heritage. She regularly visits Jokkmokk, a town above the arctic circle, which is famous for a traditional Sámi winter market and meeting place established early in the 17th century.

This February winter day, Anna is visiting her aunt Marja in Jokkmokk, whom she will help sell handmade bracelets of pewter thread and reindeer skin at the market. This is an excellent occasion for Anna to learn Sámi stories and yoiks (songs), as Marja is known for her clear voice and keen memory. At the market, Anna notices a poster that describes the mobile app for collectively building annotated archives of songs and stories, such as yoiks. Anna asks Marja if she knows about it, which Marja says she does and that it is linked to the archives at Ajtte, the Sámi museum in Jokkmokk.

Anna downloads the app after the first market day and explores it. She adds yoiks as an interest and browses some of the publicly available yoiks. She listens to a few of them and are asked a few reflective questions by the app. When she arrives to the market in the morning, her smartphone vibrates, and she notices that the app has identified yoiks related to the Jokkmokk Winter Market. One of the yoiks is from the beginning of the 20th century. Anna is very intrigued and replays the yoik for Marja to learn more about the yoik and her heritage.

Marja laughs merrily and tells Anna that it is her grandmother's uncle PerAnte that sings the yoik about his visit at the market one exceptionally cold winter. Marja tells her that PerAnte was a bear hunter and that he was widely known for his bear hides at the market plus that his wife Sara also sang yoiks. To add information in the app about PerAnte's yoik becomes their joint task during the slower periods at the market, such as adding stories of PerAnte the bear hunter, his wife Sara and their life together. They use the app to also find images to associate with the yoik, such as of items exhibited at the Ajtte museum and a museum in Berlin. Marja has an old photograph at home of PerAnte, Sara and their children in front of a goathi (a traditional Sámi tent), which they later store using the app.

Marja has learnt PerAnte's yoik when Anna returns the following summer and they agree to use the app to capture and store a video of Marja singing the yoik in front of the remains of the goathi at the outskirts of Jokkmokk. When they store the yoik they are asked about the copyrights and Marja chooses to make it publically available, but not commercially free. Two years later, Marja is contacted by a producer that wants to use her yiok as a theme song for a film about the region, inspired by the story of PerAnte and Sara. The mobile platform marketplace, that is associated with the app, have made it simple to find and use the stored yioks while taking both private and commercial considerations into account. Marja accepts and are then also invited to play a role performing the yoik in the beginning of the film.

The story of PerAnte and Sara, through the efforts of Anna and Marja, first becomes known as a good example of documenting and promoting Sámi heritage. The story is then used by the Sámi community to show how hunting rights have belonged to the Sámi, and the value thereof for the heritage. The Sámi community is in minority in all four nations, but their joint Sámi council will be able to leverage of the press coverage and public opinion to improve the conditions for the Sámi community.

These three cases reflect a diversity of spaces, from the small scale of a historic city structures (Patios) to a medium scale of historic urban areas and riverside cities 
(Lisbon), and the large scale of outdoors, green fields and forest landscapes (in the case of Sápmi). Further examples are discussed by Smaniotto et al. (2018).

\section{Theoretical Framework and User Engagement}

Cultural heritage is a key factor of European identity. Europe is a polyphonic society and its cultural wealth and advantage stem from the safeguarding and continuity of this diversity, which considers the pluralities of minorities (Pratt 2005). Societal changes affect the perception of cultural heritage by European citizens. Rapid urbanization, migration, wars and economic challenges impact European territories with evergrowing plurality of cultures and identities that must now adapt to a new concept of European citizenship. The importance of space for the preservation and communication of histories and identities has been long recognized. This understanding builds on the premise that space and landscapes contribute to the formation of local cultures and they are the framework of European natural and cultural heritage, contributing to human well-being and consolidating European identity (ELC). Co-safeguard, co-reflection on and transformation of these commons (e.g., heritage assets) can arguably contribute to the emergence of feelings of stability, continuity and belonging for people (i.e., new and existing inhabitants).

Manovich (2001: 193) argues that: "in the information age narration and description has changed roles. If traditional cultures provided people with well-defined narratives (myths, religion) and little 'stand-alone' information, today we have too much information and too few narratives which can tie it all together." The idea behind this concept is to utilise a framework of spatially - and contextually - organised narratives that relies on semantics and machine learning in order to facilitate the analysis and integration of everyday experiences, and the evolving memories of users into relevant digital cultural heritage assets. It envisions to achieve this by means of: (a) multi-user contributions, e.g., software that enables the following operations: mix, edit, reuse, enhance and enrich data features; and, (b) exchange of information, combined with expert input, guidance, reflection and discussion (Fig. 1). This data lifecycle in order to function requires the engagement of the three types of actors: (1) cultural operator, who will classify digital assets based on dedicated ontologies for each case, (2) expert users (cultural bearers), who will contextualize digital assets based on semantic models, and (3) common users, who will reflect on digital assets and add related meaning. This can be seen as an incremental and iterative process, where machine learning is applied for a high grade of automation and where these three main actors participate as part of a crowd-sourcing methodology spurred by gamification techniques. Additional actors will also be supported, such as the researcher (operating on the ontologies and algorithms) and the administrator (administrating and upgrading the platform).

The cellular structure illustrated in Fig. 2 is based on modules for Big Data Management (handling digital assets and associated metadata), the proposed platform (integrating and adapting existing tools), Machine Learning (techniques for reasoning on metadata through semantic analysis), and enabling techniques (gamification, crowdsourcing, Arianna, and social analysis), in order to facilitate the study of sociocultural representations, and participant memories and interpretations that require the 


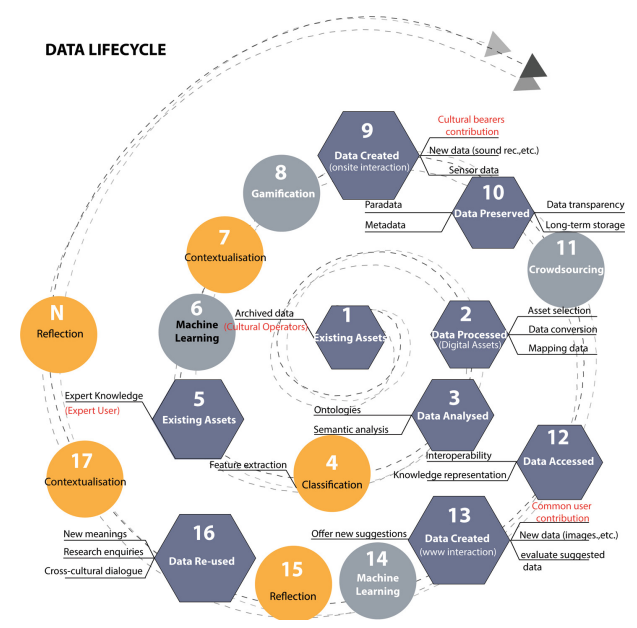

Fig. 1. Data Lifecycle. The exponentially iterative process of user engagement-collectiondigestion-semantic classification-automated suggestion loops facilitates the enrichment of data collections and reflecting on them, and thus promotes dialogue between user communities.

various groups of actors to operate feed and steer them. The interaction between the supporting ICT and each group of actors (cultural operator, expert users and common users) is made possible through participatory development methods (e.g., FormIT methods ${ }^{1}$. The practical results of this interaction are illustrated in Fig. 1, in particular the iterative and incremental augmentation of digital assets with metadata in steps 1-59-13-16. Through the proposed technological and enabling solutions this iterative process is envisioned to become catalyst in the exponential engagement of ever larger groups of users. The use of state-of-the-art technologies, such as machine learning, and novel combination of participation and engagement methods, such as co-creation, crowdsourcing and gamification, not only responds to the needs of local communities, but also promotes formation of new communities, around special cross-thematic topics (e.g., semantics, metadata management, machine learning and gamification), and facilitates drawing links between digital assets of intangible heritage and their relevant tangible heritage.

The ICT platform should consider the following:

1. Digital assets, which describe an ICH (audio tracks, video clips, photos and text notes). These digital assets will be enhanced by the (1) quantitative metadata taken automatically from the smart device: location (where), timestamp (when), user profile (who), the info coming from the sensors integrated into the smart device such as the accelerometer, gyroscope, barometer, magnetometer or light intensity (how); and (2) Qualitative metadata inserted by different users' level such as keywords (what). The digital assets will be grouped, related within a hierarchy, and subdivided according to similarities and differences through a top-level ontology

\footnotetext{
${ }_{1}^{1}$ www.ltu.se/cms_fs/1.101555!/file/LivingLabsMethodologyBook_web.pdf.
} 


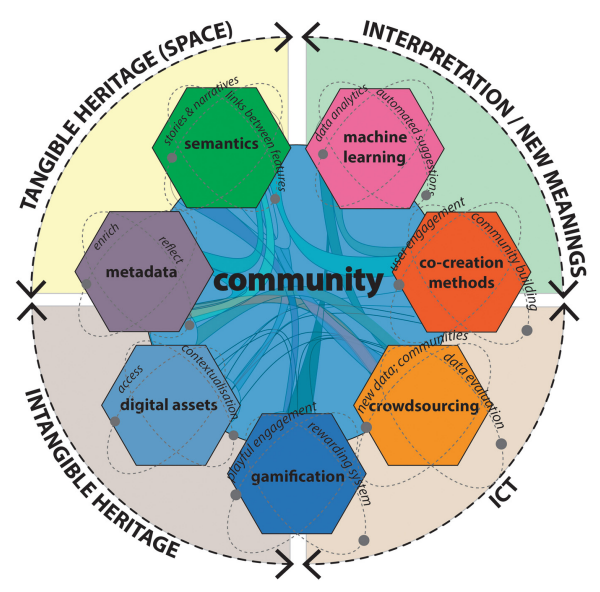

Fig. 2. Community building is the motive force of the research. By enabling this process, this platform exploits the capacity of disparate existing digital assets and local communities in order to document, collect, analyse and communicate users' interpretations of European heritage.

suitably expanded to cover the selected parts of the cases' domains. Note that localisation of ICH assets can be obtained by a number of many methods and techniques, such as automatically by Bluetooth (Nilsson et al. 2003) or user interaction with maps.

2. Users' level, each one playing different roles into the platform as explained above in data lifecycle: the cultural operator, who will provide the 1 st metadata level, i.e., the museum that owns a fado song; the cultural bearer, who will provide the 2nd metadata level, i.e. a fado singer; the common, non-expert user, who will provide the 3rd metadata level, i.e., the visitor who will enrich the ICH with own narratives; the researcher who will interact with the ontology and machine learning; and the admin who will administrate and develop further the platform.

The ICT platform, available on cloud and accessible through computers and mobile applications, consists of the following main components:

1. A mobile application, as the main interface between the space and the application server. Through this mobile app a user can upload new digital assets, insert comments on previous uploaded digital assets, reproduce them, and map dynamic information on relevant site visual representations.

2. The website, as the main portal for crowdsourcing activities (Kontkanen et al. 2016) which integrates digital assets creation and visualisation tools. The web-based access GUI enables remote visitors to become users of data and metadata available for crowdsourcing applications that will drive not only dissemination policies of the cultural players involved (e.g., Museums, collections and archives) but will also empower the system's analytical capacity in research with the power of the many.

3. The machine learning algorithm which will produce suggestions and recommendations to the users based on different criteria according to the semantic structure of the digital assets. 
4. The database, which deals with the development and execution of architectures, policies, practices and procedures that properly manage the full data lifecycle needs of the ICT platform (Fig. 1).

\section{An Innovative Approach to ICH Interpretation}

The most current need of researchers and scholars operating in the field of digital cultural heritage is how to produce quality from quantity, how to devise critical methodologies that produce meaning and generate knowledge out of big data. The process of interpretation is cross-disciplinary in nature and involves various faculties of human activity that rely on data processing, such as logical reasoning, associative analysis, descriptive capacity, linguistics and semiotic processes, decoding, and therefore cognition, abstraction and visualization, in order to reveal patterns and narratives, address the whole and provoke affection. After more than a decade of largescale digitization processes spurring from most museum, libraries and archives, the next big challenge that all cultural heritage stakeholders are facing is to make sense, to add value and establish methods of interpretation that are common, comprehensive, sharable and easily applicable to the vast archives of data and complexity of digital assets in big data.

Existing digital platforms foster collaborations among specialists instead of promoting transdisciplinary research, e.g., researchers in museums, collections, cultural and social institutions and organizations are usually establishing intra-institutional collaborations, e.g. the H2020 e-Infrastructures Project Virtual research environment for regional interdisciplinary communities in Southeast Europe and the Eastern Mediterranean ${ }^{2}$. Even more so $\mathrm{CH}$ user communities point to the strong need for tools that will enrich the capacity of researchers to utilize technological advances to move beyond digitisation tools and storage of data, seeking for new tools that will enable them to address critical interpretation problems of $\mathrm{ICH}$, an area of research that due to its context is highly transdisciplinary but has yet to invest in the digital in order to transform its models and practices. Mobile app platforms and services already available on the market include: Detour ${ }^{3}$ is a GPS-enabled audio guide that contains audio clips for historical or pop culture spots around the globe. It can sync with other smartphones that run the application so that many people experience the same audio tour at the same time; Guidekick ${ }^{4}$ is built for the San Francisco Bay Area's top historical destinations with fully interactive 3D maps, music, and narrative clips. Figure 3 illustrates a possible feature of the interface envisaged by the concept using the ICH Patios de Cordoba.

The CyberParks Songs and Stories concept builds on existing repositories (provided by the cultural operators of each case of $\mathrm{ICH}$ ) to offer an innovative combination of automation (using machine learning methods) and crowd-sourcing (user

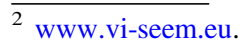

3 www.detour.com.

4 www.guidekick.co.
} 


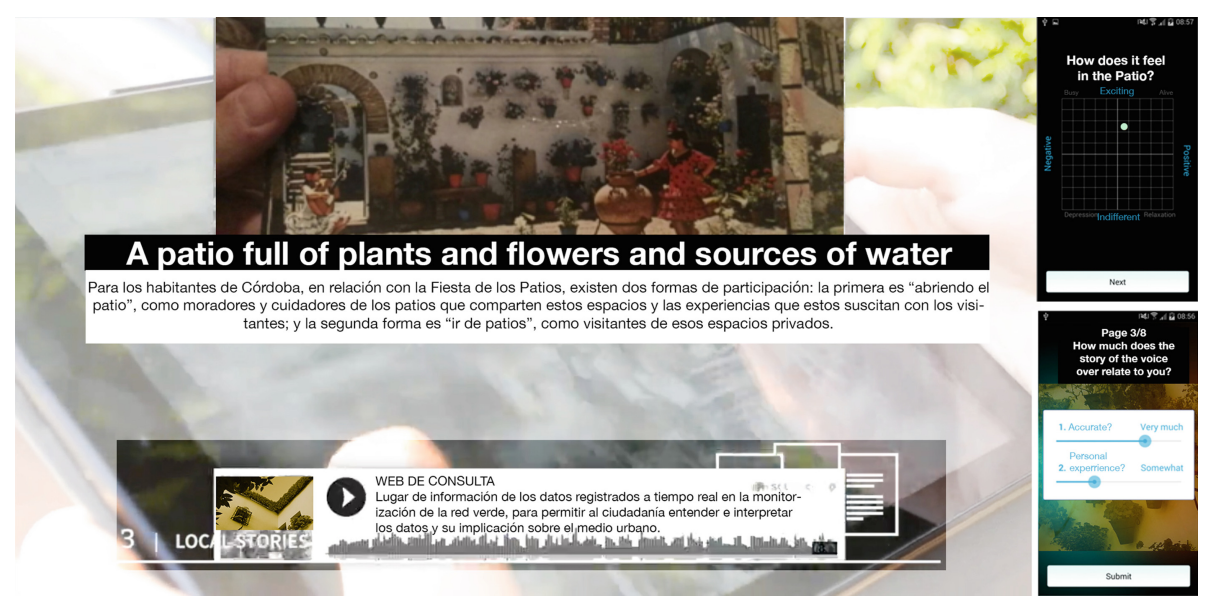

Fig. 3. A possible interface for user interface with existing digital assets and user-input data, such as a voice-over describing aspects of the community about the Patios, and a sketch of a GUI for collecting experiential data by the users of the mobile app [right column].

contextualisation and reasoning), combining machine and human processing. This effort is innovative in several ways:

1. Ensuring social inclusiveness to the data-knowledge-space continuum in heritage. Through walking, individuals interfere with physical features and obstructions, disruptions of movement, points of stasis and unpredictable situations in space, moments that intensify lived experience. The platform captures and map users' interest in heritage, and through this process, enables in-depth analysis of heritage sites' management, everyday use and occupation along with its associated cultural identities. The Living Lab methodology (FormIT) and its co-creation tools, canvases and templates offer opportunities for knowledge transfer between disciplines and user communities (e.g., cultural institutions workers, academic researchers, the creative industry and social groups). The concept innovates in combining participatory techniques with advanced digital tools in order to promote social innovation and engage individuals and groups related with specific ICH in the development of these tools - contributing to local communities feeling ownership of the tools and therefore reaching better stakes in the sustainability of the platform. The experiences of similar projects have indicated that social innovation can benefit from the integration of local community knowledge, while participatory process can contribute to enhance the self-confidence and organisational structures of local communities. Sustaining and enhancing the impact of the approach in the long run requires developing or identifying institutions that will perform work after the completion of such project, actively engaging the community as a whole and particularly young people in managing the tool and working on the profitability of the use of the tool (Zoumides 2017).

2. Crowd-sourcing for adding new meanings. The added value of the proposed concept is that it will enable the analysis and comparison of informal narratives as 
personalised interpretations (i.e., meanings). The experts' descriptions of those historical activities enriched by the (new) narratives will be expanded to include pluralities and variations that up until the present there were no tools to document and process. The enrichment of experts' knowledge with informal understandings and memories of cultural bearers who share a dual interest, not only in the specific cultural heritage but also in its associated space, can contribute to increase the understanding of European heritage.

3. Gamification for collecting interpretations and meanings. Gamification (through usage of badges, scoreboards, etc.) and playful engagement of users through storytelling, created by heritage experts rather than the entertainment industry, as in typical tourism-orientated mobile apps, and exploitation of collective intelligence through the introduction of a rewarding system for users that exploits the potential of gamification. The concept will have to give incentives to all types of users, those who add data (and metadata); who explore, assess data (and metadata) and offer suggestions to enrich them; and who exploit the platform. Playful user engagement can have multiple modes of interaction, described as they will be offered to common users inviting them to explore the associated knowledge or enrich it with their own experiences: Interactive stories, soundscapes, guided tours, audio adventure games, scavenger hunts, etc.

4. Machine learning for knowledge sharing and advanced searching. Automated semantic classification, machine learning for data analytics and personalized suggestions, and crowd-sourcing in service of heritage studies. A telling example of this innovative approach is the case of users searching in the proposed platform for a specific keyword related with a popular Fado song and being led by the automated suggestion feature of the platform to discover a relevant event to links behaviourally or thematically to the ICH of Patios - and this association facilitates the users to understand the topic they queried for from another point of view. Today changes in education and in society place new demands in learning process. Students are expected to become autonomous learners in order to self-discover knowledge rather than memorizing static information. They are asked to adopt more collaborative and critical approaches to learning than before. The discussed concept can function as a didactic platform addressed to the public that would make complex, otherwise unattainable, knowledge accessible by opening up the educational process to communities of the city that may be excluded, thus responding to the call for lifelong learning.

\section{Discussion}

The concept introduced is highly inter- and multi-disciplinary ranging from the consideration of social inclusion and gamification aspects to the application of crowdsourcing and machine-learning techniques. The belief is that the societal impact would be large if such a system would be employed for enriching public spaces with localized culture heritage material. The impacts could be: 
1. Widening the access to digitized songs and stories, to which today inherently limited to archives and museums. The potential of (mobile) Internet access to more effectively spread cultural content and knowledge is therefore huge.

2. The meta-data collected through multiple annotation processes (expert assessments, crowd-sourcing and machine learning) furthermore increase the searchability of the digitized songs and stories. Content will now be able to be match to users' context and situation as well as be possible to match with keywords expressed in an ontology structure.

3. Enriching the digitized songs and stories will furthermore add value to users. For example, digitized songs and stories can be provided with additional information and related content such as users' own versions based on the archived content.

All in all, ICH digital assets such as digitized songs and stories can thus reach a much wider audience and interest, promoting cultural heritage and its place in the modern European society. The connections between the ICH digital assets and public spaces are very important, as they provide an effective context for identifying and promoting cultural heritage. From a technical perspective, the most prominent novelties lye within the systemization of such a solution, but there are particular challenges identified:

4. The data management of the ICH digital assets is complex, as they can be stored in numerous types of archives and have various access rights. Creating one open and homogeneous system for access to the ICH digital assets are therefore a huge challenge.

5. In addition, user created content used to annotate ICH digital assets in archives are often of a more private nature, such that personal integrity issues need to be carefully considered. Users must be adequately informed; their consent retrieved and the access control for many types of applications must be provided (including social networks).

6. Perhaps the most challenging technical aspect is to jointly utilise expert knowledge, crowdsourcing and machine learning in conjunction with ontological frameworks to effectively link high quality metadata to ICH digital assets.

7. User involvement is key to the success along with applications and interfaces that support societal processes related to the use of cultural heritage. The creation of APIs for third party developers and the involvement of public organisations, such as related to public places, are therefore an important challenge.

The technologies utilised to expose cultural heritage through open access to ICH digital assets are expected to foster the development of a more inclusive and considering society that bring forth the strength of the multicultural Europe to challenge the trends of growing ultra-nationalism in many countries. 


\section{Conclusions}

This chapter addresses a potential contribution of an innovative methodology for promoting intercultural dialogue beyond Euro-centred views and assumptions by enriching European cultural expressions by means of new knowledge on heritage. It is expected that this practice will create mechanisms that collect and diffuse the cultural assets, e.g. of those who are not established cultural authorities or even by marginalized categories. Through this process it is envisioned that it will safeguard European patrimonies through everyday use of ICH digital assets that offer new ways to enhance the understanding of cultural heritage. Concluding, this research aspires to build intercultural bridges, by offering tools to foster intercultural dialogue, focused on spatiallybound ICH, on immaterial heritage associated with specific locations, to construct an inviting idea of immaterial patrimony in Europe. The technological challenges include how to manage and provide (open) access to ICH digital assets, considering the rights associated to these, how to produce meta-data and annotations to increase quality and searchability, and how to engage users through third party applications.

\section{References}

Artopoulos, G., Bakirtzis, N.: Post-digital approaches to mapping memory, heritage and identity in the city. In: Caldwell, G. (ed.) Digital Futures and the City of Today, pp. 139-156. Intellect Books, UK (2016)

Artopoulos, G., Synnes, K., Bahillo, A., Smaniotto Costa, C., Rebernik, N.: Use of data analytics for enriching public spaces with unique experiences of localised cultural heritage content. In: Busch, C., Kassung, C., Sieck, J. (eds.) Kultur and Informatik: Hybrid Systems, pp. 99-112. VWG, Glückstadt (2018)

ELC-European Landscape Convention (preamble) - European Treaty Series - Doc. No. 176

Kontkanen, J., Kärkkäinen, S., Dillon, P., Hartikainen-Ahia, A., Åhlberg, M.: Collaborative processes in species identification using an internet-based taxonomic resource. Int. J. Sci. Educ. 38, 96-115 (2016)

Manovich, L.: The Language of New Media. MIT Press, Cambridge (2001)

Pratt, M.L.: Arts of the Contact Zones, Profession 91. Modern Language Association, New York (1991, 2005)

Smaniotto Costa, C., Artopoulos, G., Djukic, A.: Reframing digital practices in mediated public open spaces associated with cultural heritage. J. Commun. Lang. 48, 143-162 (2018)

Stiegler, B.: Our ailing educational institutions: the global mnemotechnical system. Cult. Mach. 5 (2003). http://www.culturemachine.net/index.php/cm/article/viewArticle/258/243

Zoumides, C.: Community-based rehabilitation of mountain terraces in Cyprus. Land Degrad. Develop. 28, 95-105 (2017)

Nilsson, M., Hallberg, J., Synnes, K.: Positioning with bluetooth. In: 10th International Conference on Telecommunications, ICT2003, pp. 954-958 (2003) 
Open Access This chapter is licensed under the terms of the Creative Commons Attribution 4.0 International License (http://creativecommons.org/licenses/by/4.0/), which permits use, sharing, adaptation, distribution and reproduction in any medium or format, as long as you give appropriate credit to the original author(s) and the source, provide a link to the Creative Commons license and indicate if changes were made.

The images or other third party material in this chapter are included in the chapter's Creative Commons license, unless indicated otherwise in a credit line to the material. If material is not included in the chapter's Creative Commons license and your intended use is not permitted by statutory regulation or exceeds the permitted use, you will need to obtain permission directly from the copyright holder.

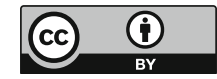

